This is a postprint version of the following published document:

Martín, O., Gutierrez, H. R., Maroto-Valiente, Á., Terrones, M., Blanco , T. \& Baselga, J. (2013). An efficient method for the carboxylation of fewwall carbon nanotubes with little damage to their sidewalls. Materials Chemistry and Physics, 140 (1-2), pp. 499-507.

DOI: 10.1016/j.matchemphys.2013.03.060

(C) Elsevier, 2013

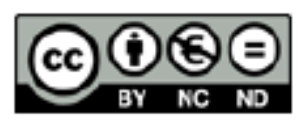

This work is licensed under a Creative Commons Attribution-NonCommercialNoDerivatives 4.0 International License. 


\title{
An efficient method for the carboxylation of few-wall carbon nanotubes with little damage to their sidewalls
}

\author{
Olga Martín ${ }^{a}$, Humberto R. Gutierrez ${ }^{b}$, Angel Maroto-Valiente ${ }^{c}$, \\ Mauricio Terrones ${ }^{d, e}$, Tamara Blanco ${ }^{f}$, Juan Baselga ${ }^{a, *}$ \\ a Department of Materials Science and Engineering and Chemical Engineering, Universidad Carlos III de Madrid, Av. Universidad 30, 28911 Leganés-Madrid, Spain \\ $\mathrm{b}$ Department of Physics and Astronomy, 102 Natural Science Building, University of Louisville, Louisville, KY 40292, USA \\ C Departamento de Química Inorgánica y Química Técnica, Facultad de Ciencias, UNED, C/ Senda del Rey 9, 28040 Madrid, Spain \\ $\mathrm{d}$ Research Center for Exotic Nanocarbons (JST), Shinshu University, Wakasato 4-17-1, Nagano 380-8553, Japan \\ e Department of Physics, Department of Materials Science and Engineering and Materials Research Institute, The Pennsylvania \\ State University, 104 Davey Lab., University Park, PA 16802-6300, USA \\ ${ }^{f}$ Materials and Processes Department, Airbus Operations S.L., Paseo John Lennon s/n, 28906 Getafe-Madrid, Spain
}

\begin{abstract}
Highlights
We report an efficient method for acid functionalization of carbon nanotubes. The method produces uniform and stable suspensions of carbon nanotubes in water.

The surfaces of the tubes are not damaged by the treatment.
\end{abstract}

\begin{abstract}
We report a novel method that is able to efficiently functionalize carbon nanotubes (few-walled: from 1 to 6 layers and multiwalled) with a high yield of carboxyl groups, based on treatments with $\mathrm{H}_{2} \mathrm{O}_{2}$ in the presence of UV light. The amount of carboxylic groups was quantified by X-ray photoelectron spec-troscopy and back-titration, showing both measurements reasonable agreement. According to the zeta potential values and to the amount of suspended nanotubes, we demonstrate that the method is able to produce uniform and stable suspensions of carbon nanotubes in water. With the aid of scanning and transmission electron microscopy, thermogravimetric analysis, and Raman spectroscopy, we show that the surfaces of the tubes are not damaged by the treatment and that the functionalized tubes have an enhanced reactivity toward oxygen. This route is efficient and could now be used to fabricate polymer composites using few-walled and multiwalled carbon nanotubes.
\end{abstract}

\section{Keywords}

Polymers Composite Materials Magnetic Materials Nanostructures Electrical Conductivity Dielectric Properties

\section{Introduction}

Carbon nanotubes, either single- (SWCNT) or multi-walled (MWCNT), exhibit extraordinary mechanical [1] and electronic $[2,3]$ properties that depend upon their chirality and degree of crystallinity. Due to their robustness and high conductivity (thermal and electrical), carbon nanotubes (CNT) could be used in the fabrication of novel composites that could lead to various types of devices including sensors [4,5]. Despite these outstanding properties of CNT, it is important to emphasize the importance of surface functionalization (e.g. carboxylation) in order to efficiently transfer their properties to different matrices (polymer, ceramics and metals).

The surfaces of highly crystalline CNTs (single- or multi-walled) interact strongly by van der Waals forces that result in the agglomeration of these tubes so as to form bundles or agglomerates [6]. These agglomerates make difficult the tube dispersion in various liquid media. This tube clustering (or bundling) is probably the main difficulty during composite preparation. The usual way to disentangle these aggregates is by modifying the crystallinity of the sidewalls, which results in weaker van der Waals interactions among tubes, using different processes that include [4]: high power ultrasonic treatments, ball milling, three roll milling, extrusion, chemical treatments, etc. [7]. 
Regarding the chemical functionalization of CNTs, various strategies have been recently used: 1,3-dipolar cycloaddition of azomethine ylides [6-6'] and adsorption of pyrene-containing polymer chains [8]. However, the most common functionalization route occurs by the generation of reactive side groups (carboxylic acid or amino groups $[9,10])$ that are responsible for disrupting the tubes' curvature and result in weaker van der Waals interactions [11]. Once the $\mathrm{sp}^{2}$-like hybridization of the carbon atoms in the tubes has been transformed into more $\mathrm{sp}^{3}$-like, efficient sidewall reactions with polymer chains could be observed so that enhanced load transfer could occur from tubes to polymers within composites.

Nanotube functionalization not only has beneficial effects on the physico-chemical interactions established between CNTs and polymer matrices, but also could result in enhanced electrical conductivity of CNT polymer composites [12-18]. In this context, carboxylic groups within graphene domains reduce the binding energies of $\mathrm{C}=$ $\mathrm{C}$ double bonds and could therefore increase the electrical conductivity of nanotube thin films. Parekh and col. [12] have observed that CNT thin films improved conductivity after $\mathrm{HNO}_{3}$ oxidation and $\mathrm{SOCl}_{2}$ contact. Wang et al. [17] studied the effect of the carboxylic functionalization of SWCNT using the density functional theory, and they found that the electrical conductivity would be significantly improved by increasing the axial density of $-\mathrm{COOH}$ groups.

CNT functionalization involving partial oxidation processes has been reported by numerous researchers [19-34]. For example, Liu et al. [20] first reported the severe oxidative treatment of SWNT when using a reflux mixture of concentrated sulfuric and nitric acids $\left(\mathrm{H}_{2} \mathrm{SO}_{4} / \mathrm{HNO}_{3} 3: 1,98 \%\right.$ and $40 \%$ respectively). Interestingly, variations of this method have been widely used by various researchers to purify (removal of catalyst impurities and amorphous carbon) and functionalize SWCNT and MWCNT [16,17,20,21,24]. González-Guerrero et al. [25] found that the concentration of total acidic groups increased up to $2.5 \times 10^{-5} \mathrm{~mol} \mathrm{mg}^{-1}$ after $6 \mathrm{~h}$ of refluxing time, and remained constant at longer refluxing periods. The oxidative purification of catalytically prepared nanotubes by $\mathrm{KMnO}_{4}, \mathrm{H}_{2} \mathrm{O}_{2}, \mathrm{HClO}_{4}$ and ozone has also been reported [31]. The results indicate that $\mathrm{KMnO}_{4}$ creates an oxidized tube that is free from amorphous carbon; however, additional steps are needed to remove the $\mathrm{MnO}_{2}$ that is generated during the oxidation process. Kuznetsova et al. [32] have demonstrated that both carbonyl and ether groups are produced when SWNTs are purified using $\mathrm{H}_{2} \mathrm{SO}_{4} / \mathrm{HNO}_{3}$ and $\mathrm{H}_{2} \mathrm{SO}_{4} / \mathrm{H}_{2} \mathrm{O}_{2}$ mixtures. The effect of partial chemical oxidation of SWNTs using various oxidizing agents has also been reported by Yang and co-workers [19]. These authors indicate that various functional groups can be introduced to the tubes surfaces when the SWNTs are treated with different oxidizing agents. Dilute nitric acid has shown to generate carboxylic acid groups on defect sites already present within nanotubes, whereas a mixture of concentrated sulfuric and nitric acids generates carboxylic acid groups on newly created defective sites $[23,24]$. Potassium permanganate introduces different amounts of hydroxyl, carbonyl and carboxylic groups within the nanotubes surface [35]. But all these methods generally have low yields and extensive post-treatment purification processes are usually needed to remove traces of unwanted chemicals. In addition, some of these methods are very aggressive and the surfaces of the tubes could get severely damaged creating defective sites, reducing their strength and changing therefore their size or aspect ratio.

It is well known that oxidative processes involving $\mathrm{sp}^{2}$ hybridized carbon atoms start with the creation of an alcohol group, followed by a ketone group, and finally yielding a carboxylic group [20]. The next oxidative step in a high oxidizing environment involves the generation of $\mathrm{CO}_{2}$, which is released from the structure thus creating "holes or vacancy defects" within the graphene layer. However, chemical treatments with concentrated acids could seriously affect the overall crystallinity of the tubes by the introduction of an excessive number of surface defects. Hence, some researchers have studied alternative milder methods such as ozone oxidation [27-29,36]. These methods have found that ozone or a combination of UV-ozone or water vapor, generate oxygenated functional groups such as $\mathrm{C}-\mathrm{OH},-\mathrm{COOH}$ and $-\mathrm{C}=\mathrm{O}$ on the sidewalls of CNTs through free radical formation. In the same way, Li et al. [30] reported oxidation of MWCNT using Fenton's reagent, and showed that MWCNTs could be successfully functionalized with hydroxyl radicals which could be reactive all along the tube walls.

Therefore, alternative routes to develop a high yield carboxylation process without damaging the sidewalls of CNTs need to be discovered. In this paper, we report a safe route that combines ultraviolet light able to generate radicals, coupled with a mild oxidant, $\mathrm{H}_{2} \mathrm{O}_{2}$. We also report and test this simple and efficient method for high yield carboxylation of few-walled carbon nanotubes (FWCNT), a generic name for nanotubes with low number of walls (e.g. double-, triple- or four-walled), which also results in a low degree of damage of the sidewalls. The resulting functionalized FWCNT have been studied by X-ray photoelectron spectroscopy (XPS), transmission electron microscopy (TEM), scanning electron microscopy (SEM), thermogravimetric analysis (TGA), Raman spectroscopy, elemental analysis, back titration and $\zeta$-potential measurements. In addition, we have confirmed that the proposed oxidation treatment increases nanotube solubility in water. We believe these mildly treated tubes could now be used in the fabrication of polymer composites, since we envisage strong interactions of the tube walls with various types of polymer chains.

\section{Experimental}

\subsection{Materials and reagents}

Chemical vapor deposition (CVD) synthesized CNT were purchased from Shenzhen Nanotech Port Co. Ltd. (China) with a purity $>90 \%$, diameter $<2 \mathrm{~nm}$ and lengths between 5 and $15 \mu \mathrm{m}$. Analyzed samples contained a mixture of nanotubes with a number of walls ranging from 1 to 6 . According to manufacturer specification, these tubes were acid purified and this fact justifies the presence of acid groups on the surface of pristine nanotubes. To extend this study to other nanotubes from different sources and with different structure, MWCNT from Arkema (Graphistrength, GS, 10-15 nm diameter, 0.1-10 $\mu \mathrm{m}$ length) and MWCNT from Nanocyl (NCL, $9.5 \mathrm{~nm}$ diameter, $1.5 \mu \mathrm{m}$ length) were acquired. Hydrogen peroxide $30 \%$ $\mathrm{w} / \mathrm{v}$ was supplied by Panreac; water solutions of $0.1 \mathrm{~N}$ sodium hydroxide and $0.1 \mathrm{~N}$ hydrochloric acid volumetric standards were supplied by Sigma Aldrich.

\section{2. $\mathrm{H}_{2} \mathrm{O}_{2} / \mathrm{UV}$ oxidation}

In a typical process $1 \mathrm{~g}$ of as-received FWCNTs was dispersed in $25 \mathrm{ml}$ hydrogen peroxide $30 \% \mathrm{w} / \mathrm{v}$ (100 vol.) in an open culture disk beaker, immersed in an ultrasonic bath and irradiated with a UV light (Philips Lighting 250HPLN 250W without filter) at $15-20 \mathrm{~cm}$ lamp distance during $15 \mathrm{~min}$. The resulting dispersion was diluted in distilled water, cleaned and filtered several times. Before drying, the wet sample was ground for $20 \mathrm{~min}$ in an agate mortar and finally lyophilized. Nanotubes treated in this way will be called oxidized FWCNTs all along this paper in order to avoid confusion with asreceived or pristine FWCNT that also contain traces of acid groups. This method was also applied to multiwall nanotubes: GS and NCL.

\subsection{Characterization}

XPS data were recorded with an Omicron spectrometer equipped with an EA-125 hemispherical electron multichannel analyzer 
and an unmonochromatized $\mathrm{Mg} \mathrm{K} \alpha \mathrm{X}$-ray source operating at $150 \mathrm{~W}$ with a pass energy of $50 \mathrm{eV}$. The samples were pressed into small pellets of $15 \mathrm{~mm}$ diameter, mounted on the sample holder and introduced into the chamber where they were degassed for 6$8 \mathrm{~h}$ until a dynamic vacuum below $10^{-8}$ Pa prior XPS analysis was achieved. The recorded spectra were analyzed using CASAXPS software, and RSF database by peak fitting after Shirley background correction.

Metal elemental analyses on both pristine and oxidized nanotubes were carried out by XR fluorescence using the Xepos III from Spectro Analytical Systems using the TurboQuant Powders method.

SEM observations were carried out using a FEI-XL30 microscope equipped with a backscattered electron detector (BSE) and an energy dispersive analyser (EDAX D4i). Samples were prepared as follows: $0.1 \mathrm{mg}$ of CNT were ultrasonically dispersed in $10 \mathrm{ml}$ of isobutanol for $10 \mathrm{~min}$ and a couple of drops of solution were deposited on the sample holder and dried in vacuum at $60{ }^{\circ} \mathrm{C}$ for $1 \mathrm{~h}$. TEM characterization was performed in a $200 \mathrm{keV}$ JEOL $2010 \mathrm{~F}$ electron microscope. A suspension of CNT in isopropanol was prepared ultrasonically and a drop was deposited in Lacey carbon grids. Raman spectroscopy measurements of both pristine and oxidized samples were carried out in a microscope-based in Via Renishaw Raman Spectrometer, using the $514 \mathrm{~nm}$ line from an argon laser as the excitation wavelength. For each sample, several spectra were taken in different places to verify the homogeneity of the sample structure and composition.

The zeta potential values of pristine and oxidized FWCNTs were measured in aqueous solution $\left(50 \mathrm{mg} \mathrm{L}^{-1}\right)$ at $\mathrm{pH}=7.4$, using a nano Zetasizer (Malvern Instruments, UK). Titration (Schott CG842 pH meter) was carried out with distilled and boiled water in order to keep out $\mathrm{CO}_{2}$. In a typical experiment, $0.1 \mathrm{~g}$ FWCNTs were added to $15 \mathrm{ml}$ of a $0.1 \mathrm{~N} \mathrm{NaOH}$ solution, then sonicated for $1 \mathrm{~h}$ and stirred during $14 \mathrm{~h}$, to allow the solid CNT material to equilibrate with the $\mathrm{NaOH}$ solution. After this FWCNTs were removed by filtration, and the resulting material was back titrated with a $0.096 \mathrm{~N} \mathrm{HCl}$ aqueous solution.

Thermogravimetric (TGA) analyses were performed on a Perkin Elmer 6000 STA system heating from $50^{\circ} \mathrm{C}$ to $900^{\circ} \mathrm{C}$. Approximately $5 \mathrm{mg}$ of sample were heated in an open platinum crucible up to $1000^{\circ} \mathrm{C}$ at a rate of $10^{\circ} \mathrm{C} \mathrm{min}^{-1}$ under air flow $\left(20 \mathrm{ml} \mathrm{min}^{-1}\right)$. Oxygen and nitrogen contents were determined on a LECO TC500 analyzer.

\section{Results and discussion}

The chemical nature of the functional groups generated on the CNT sidewalls was examined by XPS. The C1s and O1s peaks obtained for $\mathrm{H}_{2} \mathrm{O}_{2} / \mathrm{UV}$ oxidized FWCNTs are presented in Fig. 1.

Both peaks have been fitted by deconvoluting different contributions from separate binding energies, see Table 1.

Results for pristine and oxidized FWCNTs are presented in Table 2 where it can be observed that as-received FWCNTs contain a certain amount of oxygen and carboxylic groups on their surface, probably due to the supplier purification method; but after our oxidation treatment the $\mathrm{O} / \mathrm{C}$ ratio increases by a factor of 4 . Analysis of $\mathrm{C} 1 \mathrm{~s}$ and $\mathrm{O} 1 \mathrm{~s}$ signals reveals that this excess in oxygen is mainly due to alcohol and carboxylic groups, whereas for as-received FWCNTs the highest signal corresponds to carbonyl groups. Moreover, from the $\mathrm{O} / \mathrm{C}$ ratio along with the carboxylic specific signal it can be estimated the number of carboxylic equivalents per at-g $C$, resulting in $\sim 10^{-3}$ eq (at-g C) ${ }^{-1}$ for the pristine CNT and $\sim 10^{-2}$ eq (at-g C) $)^{-1}$ for the oxidized ones. To the best of our knowledge this high carboxylation yield using a short and mild oxidation treatment has not been reported hitherto.

In order to verify these results, elemental analyses, as well as back titration experiments were performed. Results are presented in Table 3.

Back titration results indicate an increase of $65 \%$ in carboxylic groups for FWCNTs. Expressing carboxylic concentration data in
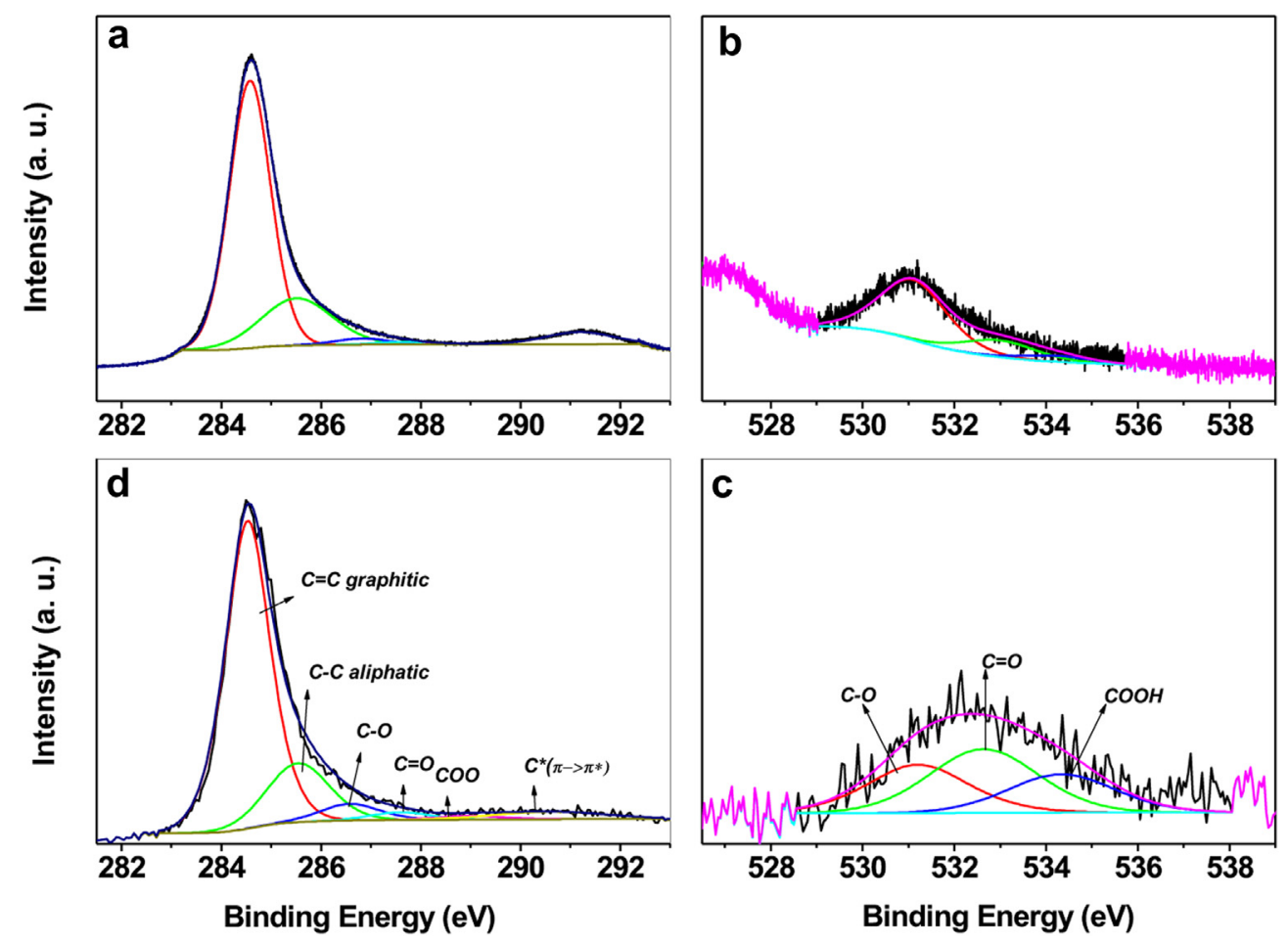

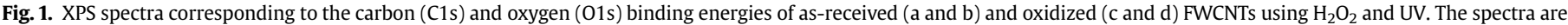

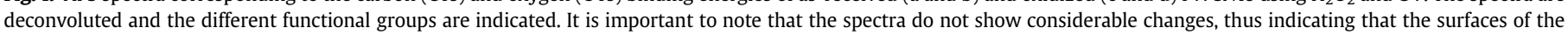
tubes were not heavily altered after the oxidation treatment. 
Table 1

Binding energy ranges $(\mathrm{eV})$ of several functional groups used for the convolution of FWCNT XPS spectra.

Binding energy range (eV)

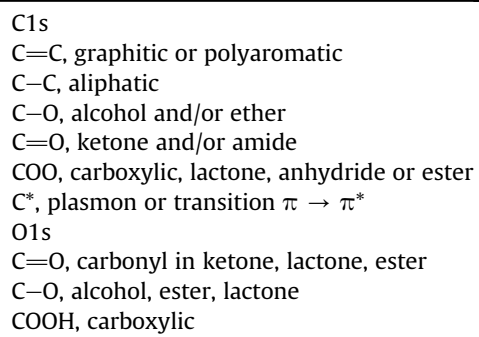

$$
\begin{aligned}
& 284.5-284.6 \\
& 285.5-285.6 \\
& 286.4-286.9 \\
& 287.2-287.8 \\
& 288.7-289.1 \\
& 290.5-291.2 \\
& \\
& 531.0-532.0 \\
& 533.0-532.6 \\
& 533.9-534.9
\end{aligned}
$$

terms of equivalents per at-g $C$ results are $2 \times 10^{-3}$ eq (at-g C $)^{-1}$ for the pristine and $3.3 \times 10^{-3} \mathrm{eq}(\mathrm{at}-\mathrm{g} \mathrm{C})^{-1}$, in fair accordance with XPS results presented above. Elemental analysis shows that the oxygen content increases $41 \%$; it should be noted that if this excess in oxygen had been used solely in generating new carboxylic groups, the increment of acidic groups would have been only $\mathrm{ca}$. $20 \%$. Therefore, these results suggest that our chemical-UV treatment generates carboxylic groups via two parallel processes: i) direct oxidation of $\mathrm{sp}^{3}$-like hybridized carbon atoms and ii) oxidation of other oxidized forms of carbon, probably from pre-existing carbonyl groups since their content decreases significantly (Table 2). To check the validity range of the method, multiwall CNTs from other sources were also oxidized. Results are also presented in Table 3, where it can be observed that the carboxylic acid content increased $23 \%$ for NCL and $50 \%$ for GS.

Luque and col. [37] studied theoretically the adsorption of carboxylic groups and hydrogen peroxide on perfect and defective (Stone-Wales, 5 or 7 rings) graphene sheets through the density functional theory. Three of their conclusions could help to understand the oxidation mechanism underlying the findings presented here at least in qualitative terms. The first one is that functionalization of graphene sheets with carboxylic acids seems to occur preferably in the defects acting as nucleation centers for successive functionalization steps, i.e. once a first $\mathrm{COOH}$ group has been adsorbed, adsorption of a second one on neighbor carbon atoms induces a strong stabilization. With regard to $\mathrm{H}_{2} \mathrm{O}_{2}$ adsorption, these authors found that the limiting process for adsorption of the $\mathrm{OH}$ species on perfect and defective graphene sheets is the cleavage of the $\mathrm{O}-\mathrm{O}$ bonds of hydrogen peroxide. Once $\mathrm{OH}^{*}$ radicals are formed they can accommodate easily on defective graphene sheets, e.g., $\mathrm{sp}^{3}$ hybridized carbon atoms, but due to the extensive reorganization of the $\mathrm{sp}^{2}$ network, this process is extremely slow in a perfect substrate. Moreover, if the surface has been previously

\begin{tabular}{|c|c|c|c|c|c|c|c|c|c|c|}
\hline & \multicolumn{6}{|c|}{ C 1s (at \%) } & \multicolumn{3}{|c|}{ O1s (at \%) } & \multirow[t]{2}{*}{$\mathrm{O} / \mathrm{C}$} \\
\hline & $\mathrm{C}=\mathrm{C}$ & $\mathrm{C}-\mathrm{C}$ & $\mathrm{C}-\mathrm{O}$ & $\mathrm{C}=\mathrm{O}$ & $\mathrm{COO}$ & $C^{*}$ & $\mathrm{C}=\mathrm{O}$ & $\mathrm{C}-\mathrm{O}$ & $\mathrm{COOH}$ & \\
\hline $\begin{array}{l}\text { Pristine } \\
\qquad F W C N T S\end{array}$ & 69.1 & 22.6 & 1.5 & 1.0 & 0.4 & 5.4 & 71.5 & 21.3 & 7.2 & 0.019 \\
\hline $\begin{array}{l}\mathrm{H}_{2} \mathrm{O}_{2} / \mathrm{UV} \\
\quad \text { FWCNTS }\end{array}$ & 70.1 & 17.9 & 4.7 & 2.2 & 1.1 & 4.0 & 31.3 & 43.2 & 25.6 & 0.081 \\
\hline
\end{tabular}
functionalized with carboxylic groups absorption of $\mathrm{OH}^{*}$ species seems to be specially favored.

Experimentally, room temperature oxidation of carbon nanotubes with hydrogen peroxide alone has been explored by Peng and col. [38]. Their results are in agreement with calculations made by Luque and col. [37]: hydroxylation takes place very slowly along

Table 2

Functional groups content on the surface of FWCNTs after deconvolution analysis. several days preferentially on network defects and in a subsequent stage, $-\mathrm{OH}$ and lactone groups transform into carboxylic groups and $\mathrm{CO}_{2}$ with removal of impurities. It seems therefore that hydrogen peroxide photolysis with UV radiation is the key process to speed up the oxidation process. It is well known that $\mathrm{H}_{2} \mathrm{O}_{2}$ can be dissociated with UV light according to the following reaction

$\mathrm{H}_{2} \mathrm{O}_{2} \stackrel{h v}{\rightarrow} 2 \mathrm{OH}^{\cdot}$

generating $\mathrm{OH}^{*}$ radicals which are strong oxidants. In fact, photo dissociation of hydrogen peroxide has been used as a general oxidation process for reducing pollutants, dyes and organic matter (see for example the work of Muruganandham [39] and references contained therein) under mild acidic conditions. The natural $\mathrm{pH}$ of pristine FWCNT water dispersions is slightly acidic, adequate to avoid loses of hydrogen peroxide by self-ionization. Through UV irradiation, the generated $\mathrm{OH}^{*}$ radicals oxidize hydroxyl and carbonyl preexisting species and, at the same time, becomes adsorbed in defect sites of the $\mathrm{sp}^{2}$ graphene sheet, although these tubes do not present a high proportion of defects as revealed by thermal analysis (see below). As carboxylic groups are formed, subsequent radical absorption should occur on the distorted neighboring carbon atoms in accord with Luque and col. [37] theoretical findings. If this mechanism operates, the distribution of carboxylic groups along the walls of the tubes should not be homogeneous but concentrated in small clusters. More work is presently being carried out to verify this point.

An alternative source of $\mathrm{OH}^{*}$ radicals may come from the iron catalyzed hydrogen peroxide decomposition, either dark or photoassisted, that may play a role because of the presence of catalyst impurities. In this process, known as the Fenton oxidation, the highest rate corresponds to the oxidation of $\mathrm{Fe}^{2+}$ to $\mathrm{Fe}^{3+}$ producing hydroxyl radicals. It requires the presence of $\mathrm{Fe}^{2+}$ ions that can be formed by oxidation of the reduced iron present in the catalyst systems. To verify if this process could operate in the functionalization of nanotubes, elemental analysis was performed before and after the oxidation treatment by XR fluorescence. Amounts of the main impurities are presented in Table 3. The presence of several transition metals is attributed to catalyst residues, and the presence of $\mathrm{Al}$ is attributed to the catalyst support. The high $\mathrm{Al}$ content found for GS and NCL suggests these systems use a Fe catalyst supported system, whereas nanotubes from Shenzhen probably use a nonsupported catalyst with Co as main component. Iron content is greatly reduced after oxidation for Shenzhen nanotubes so for these systems, Fenton oxidation is a possible route. GS nanotubes present similar iron content but it remains practically constant before and after oxidation; this may reflect that catalyst residues are not accessible to hydrogen peroxide so Fenton oxidation route can be excluded. Similarly, iron content in NCL nanotubes is roughly 10 times lower and also remains constant; if Fenton route would operate carboxylic content would have been about ten times lower and this is not observed. Therefore, it can be concluded that although Fenton oxidation is a plausible parallel route for nanotube functionalization, it is not responsible for the high carboxylic content obtained in GS and NCL systems.

In previous reports, it has been observed that high temperature oxidation treatments using $\mathrm{HNO}_{3}$ on SWCNTs may generate carbonaceous debris adsorbed on the nanotubes surface containing the great majority of carboxylic groups [40]. This carbonaceous material (also known as fulvic acid) has also been observed in oxidized MWCNTs $[41,42]$ which was then removed after a basic treatment with $\mathrm{NaOH}$, thus decreasing the amount of functional groups anchored to the CNT sidewalls. Our chemical-UV oxidation treatment is too mild to result in the generation of adsorbed fulvic 
Table 3

Elemental analysis, carboxylic content (back titration) and $\zeta$-potential of commercial and oxidized FWCNTs and other commercial multiwalled CNTs.

\begin{tabular}{|c|c|c|c|c|c|c|c|c|c|}
\hline Sample & $\mathrm{O}(\%)$ & $\mathrm{N}(\%)$ & $\mathrm{Al}(\%)$ & $\mathrm{Fe}(\%)$ & Co (\%) & Mo (\%) & $\begin{array}{l}{[-\mathrm{COOH}] \times 10^{4}} \\
\left(\text { eq. } \mathrm{g}^{-1}\right)\end{array}$ & $\Delta[-\mathrm{COOH}](\%)$ & $\zeta$-potential $(\mathrm{mV})$ \\
\hline Pristine FWCNTs & 1.83 & 0.17 & 0.03 & 2.1 & 8.6 & 0.75 & 1.7 & 65 & $-24.5 \pm 5.8$ \\
\hline $\mathrm{H}_{2} \mathrm{O}_{2} / \mathrm{UV}$ FWCNTS & 2.58 & 0.15 & 0.03 & 0.04 & 2.1 & 0.13 & 2.8 & & $-28.2 \pm 5.5$ \\
\hline Pristine GS & - & - & 4.7 & 2.4 & - & - & 0.8 & 50 & - \\
\hline $\mathrm{H}_{2} \mathrm{O}_{2} / \mathrm{UV}$ GS & - & - & 4.6 & 2.2 & - & - & 1.2 & & - \\
\hline Pristine $N C L$ & - & - & 7.7 & 0.3 & 0.09 & - & 2.6 & 23 & - \\
\hline $\mathrm{H}_{2} \mathrm{O}_{2} /$ UV NCL & - & - & 10.2 & 0.44 & 0.15 & - & 3.2 & & - \\
\hline
\end{tabular}

acids but in order to ensure that after our oxidation treatment, the generated carboxylic acids were firmly anchored to nanotube sidewalls, the oxidized nanotubes were treated with $1 \mathrm{M} \mathrm{NaOH}$ for $1 \mathrm{~h}$, filtered, extensively washed with water and back titrated again. We then observed that the amount of acidic sites remained unaltered after this treatment, indicating that acidic groups generated after our oxidation method are located on the nanotube sites and do not form part of adsorbed fulvic acids.

A direct consequence of generating carboxylic groups within the nanotube sidewalls consists of an increase of the surface potential due to ionization reactions in water. The surface electric potential could be characterized by $\zeta$-potential measurements. In this context, Kim et al. [15] reported that $\zeta$-potential of pristine nanotubes exhibited a positive value, whereas oxidized nanotubes using $\mathrm{HNO}_{3}$ were negatively charged, and the absolute value increased with the strength of the oxidation process. $\zeta$-potential values for our commercial and oxidized FWNTs are presented in Table 3. In agreement with our previous findings, both as-received and oxidized nanotubes exhibit a negative potential being lower for the oxidized nanotubes. It is noteworthy that our results show lower values than those reported by Bae et al. [43] and are similar to those found by Kim et al. [15], who used a more aggressive oxidation treatment ( $1 \mathrm{~h}$ at $100{ }^{\circ} \mathrm{C}$ with $40 \% \mathrm{HNO}_{3}$ ); however, $\zeta$-potential values are higher than those reported by $\mathrm{Hu}$ [44], who used very long treatment periods in the presence of $\mathrm{HNO}_{3}$.

An important goal of the carboxylation of CNT was to increase their dispersibility without resulting in severe structural damage. So we measured the solubility of the nanotubes in water shortly after its dispersion by a 30 s sonication treatment and 30 days after to test dispersion stability. A maximum concentration of oxidized nanotubes (132 $\mathrm{mg} \mathrm{L}^{-1}$ ) was observed after $1 \mathrm{~h}$, whereas pristine nanotubes only displayed $28 \mathrm{mg} \mathrm{L}^{-1} .30$ days after making these dispersions, it was found that the concentration of pristine nanotubes in water decreased to $0.4 \mathrm{mg} \mathrm{L}^{-1}$ but the concentration of the oxidized ones was almost ten times greater $\left(3.7 \mathrm{mg} \mathrm{L}^{-1}\right)$. Therefore, our oxidation method notably increases both the short and long term solubility.

In order to confirm the post treatment degradation state and dispersibility, TGA, SEM, TEM and Raman spectroscopy were carried out. Representative SEM and TEM images of both as-received and oxidized nanotubes are shown in Figs. 2 and 3.

Dilute dispersions of pristine nanotubes in isobutanol indicate a tendency to form bundles of tubes, but it can be clearly observed how the functionalized FWCNTs become less agglomerated and exhibit no apparent damage on the tubes walls (see Fig. 3). In some occasions, along with these tubes, some particles, probably graphitic carbon and catalyst residues, are also present.

TEM images of pristine and oxidized FWCNT samples did not reveal considerable differences in terms of purity, structure and tube wall quality. From low magnification images (Fig. 3, top) it can be noticed that the samples are mainly composed of CNTs and the concentration of catalysts nanoparticles as well as amorphous carbon is low. Higher magnification TEM images reveal that the tube walls range between one and six. These images also indicate that the walls quality and structure are well preserved after the oxidation process (Fig. 3, bottom), which is in good agreement with SEM observations and with Raman spectra which are discussed below. GS and NCL tubes were also studied by TEM; as with FWCNT, the corresponding images (Figure SI- 1 a and b, Supplementary information) did not reveal any considerable difference in terms of wall structure.

Fig. 4 shows the Raman spectra of pristine (top) and oxidized (bottom) FWCNTs; the corresponding spectra or GS and NCL MWCNTs are presented in Figure SI-2 a and b. For each sample we
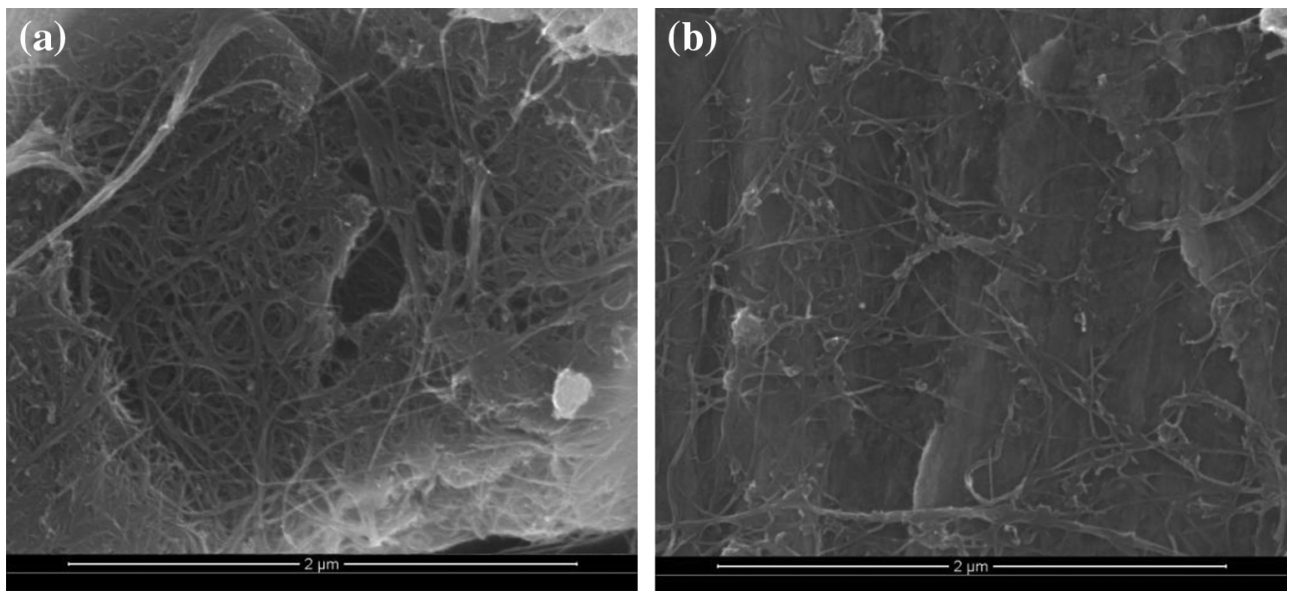

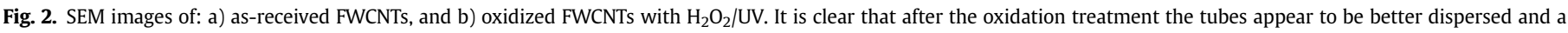
fewer number of bundles are observed. 

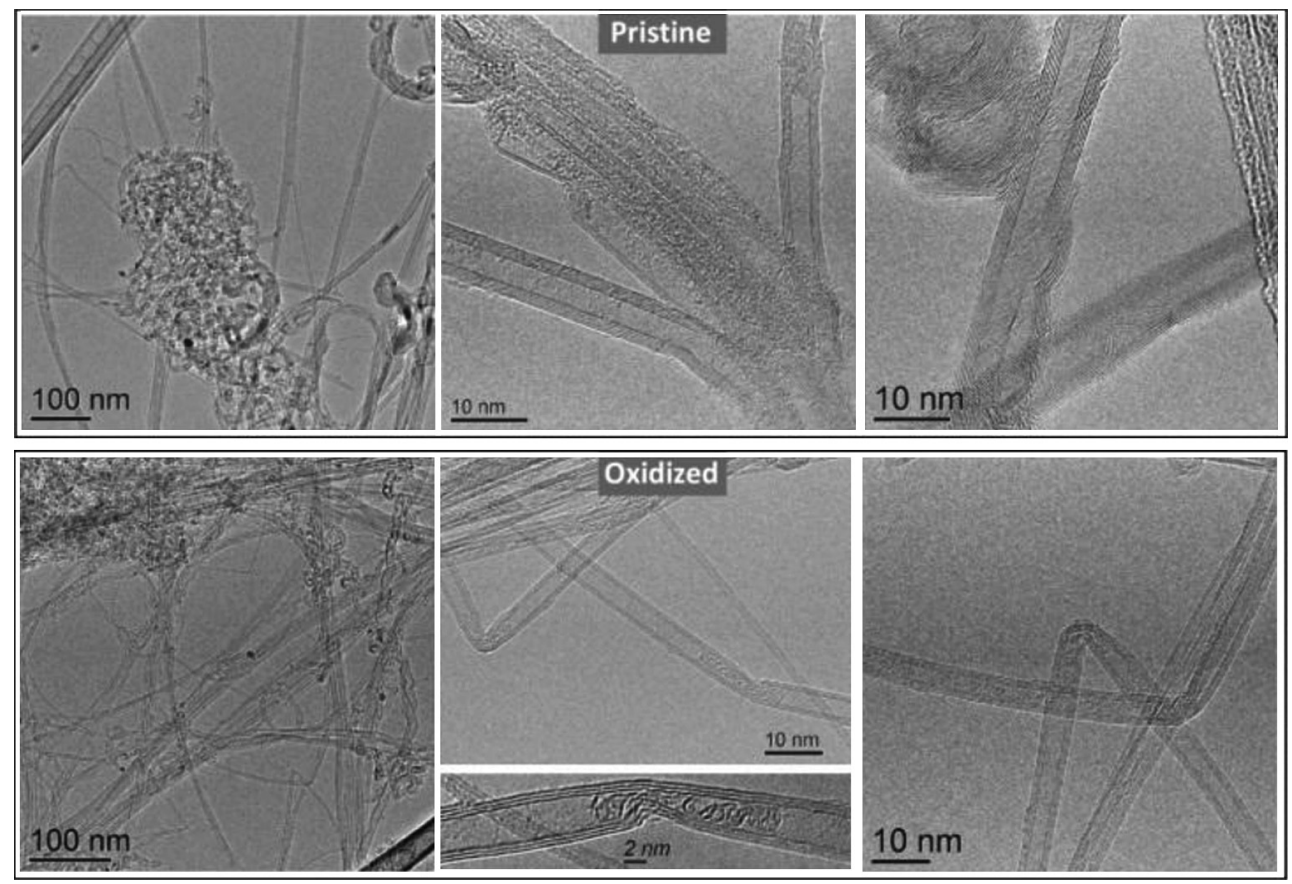

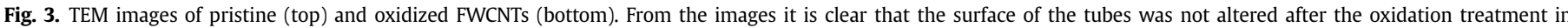
$\mathrm{H}_{2} \mathrm{O}_{2} / \mathrm{UV}$. The nanotubes exhibited from 1 to 6 walls.

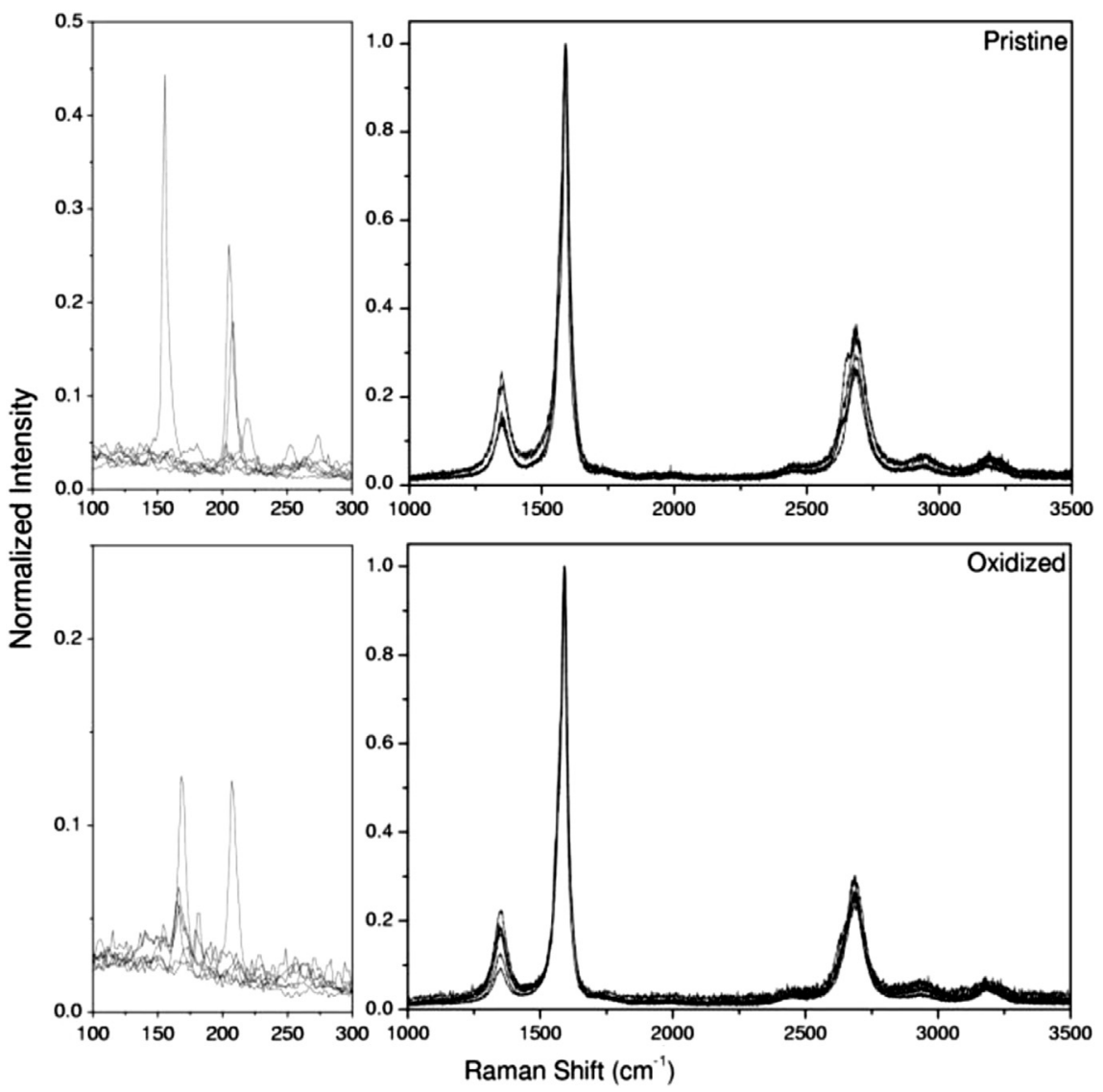

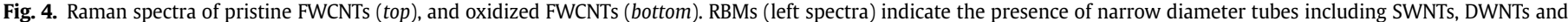

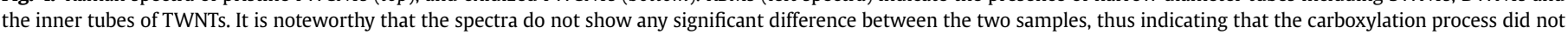
affect the surface structure of our FWCNTs. 
recorded at least eight different spectra from different locations within samples in order to verify the homogeneity of the materials. We noted that the average variations of the relative intensities as well as the position of the characteristic Raman peaks (D at $\sim 1358 \mathrm{~cm}^{-1}, \mathrm{G} \sim 1589 \mathrm{~cm}^{-1}$ and $2 \mathrm{D} \sim 2689 \mathrm{~cm}^{-1}$ ) are very similar in both samples, suggesting that the oxidation-functionalization process did not affect considerably neither the structure of FWCNTs nor GS or NCL.

The Raman peaks at lower frequencies $\left(100-300 \mathrm{~cm}^{-1}\right)$ known as Raman breathing modes (RBM) are generally observed in single-, double- and triple-walled CNTs, and its spectral position depends on the nanotube diameter. The range of RBM positions in both samples was broad and not affected by the oxidation process.

The effect of the oxidation treatment on thermal stability and thermal degradation mechanism of the tubes was analyzed by thermogravimetry. TGA curves are depicted in Fig. 5. As received FWCNTs experience a slight increase in weight up to $480{ }^{\circ} \mathrm{C}$, attributed to the oxidation of the catalyst residue. This preoxidation stage, which is absent in the oxidized samples, has been previously reported in literature by other authors that carried out the functionalization of SWNTs [45]. In the temperature range between 450 and $660{ }^{\circ} \mathrm{C}$, a sharp weight decrease occurs for both samples. Finally, a post oxidation residue is detected for both samples: $13.0 \%$ for as-received FWCNTs, and 3\% for the oxidized ones. The first value reasonably agrees with the supplier purity information (>90\%). The lower value found for the oxidized FWCNTs suggests that the carboxylation treatment partially removes unwanted residues. It is noteworthy to observe how quickly the catalyst impurities are reduced after only $15 \mathrm{~min}$ of $\mathrm{H}_{2} \mathrm{O}_{2} /$ UV oxidation.

By analyzing the first derivative of the curves from the TGA data, we noted that the curves reveal a minimum, corresponding to the maximum oxidation rate, $T_{\mathrm{m}}$, which is located at $565{ }^{\circ} \mathrm{C}$ for our oxidized material, and at $603{ }^{\circ} \mathrm{C}$ for as-received FWCNTs. As stated by Arepalli et al. [46] $T_{\mathrm{m}}$ values depend on different factors that include, tube diameter, defects, presence of catalyst impurities and derivatization moieties located on the nanotube sidewalls. Therefore, a broad range of $T_{\mathrm{m}}$ values are found in the literature. In Table 4, it is presented a brief survey of $T_{\mathrm{m}}$ data for SWCNT from different sources but similar to those used in this work.

Analysis of the data reveals that defective nanotubes contaminated with amorphous carbon present a maximum oxidation rate below $500{ }^{\circ} \mathrm{C}$ and as the nanotubes are purified and annealed to reduce the number and extent of defects, $T_{\mathrm{m}}$ increases up to $600^{\circ} \mathrm{C}$

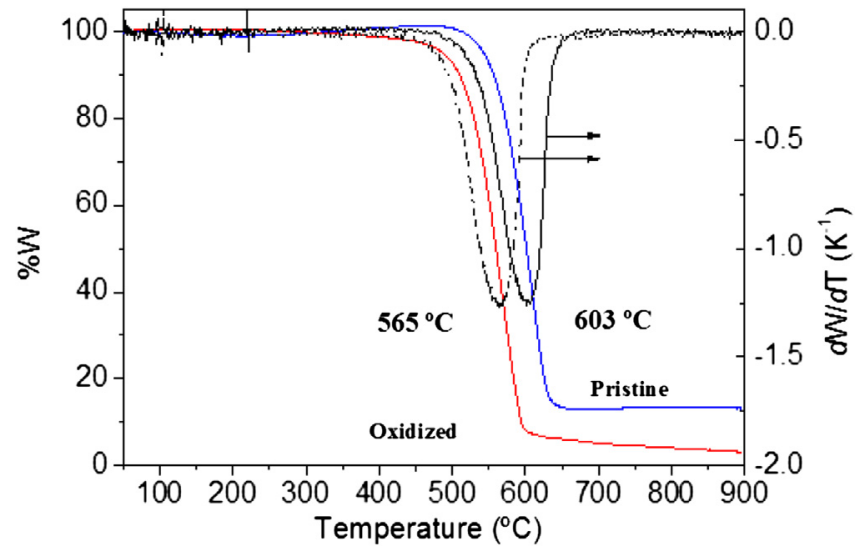

Fig. 5. TGA plots of FWCNTs: a) pristine, and b) oxidized by $\mathrm{H}_{2} \mathrm{O}_{2} /$ UV. It is clear that the oxidized material burns out at lower temperatures when compared to the pristine tubes. Therefore, the reactivity of the surface has been modified by the process and the oxidized tubes are more reactive with oxygen at higher temperatures.
Table 4

Thermal stability, $T_{\mathrm{m}}$, at $10^{\circ} \mathrm{C} \mathrm{min}^{-1}$ of several SWCNT from different sources.

\begin{tabular}{lll} 
Source & $T_{\mathrm{m}}\left({ }^{\circ} \mathrm{C}\right)$ & Ref \\
\hline Sigma-Aldrich & 380 & {$[47]$} \\
Carbon Nanotechnologies & 500 & {$[47]$} \\
Nanocyl & $514-534^{\mathrm{a}}$ & {$[48]$} \\
HiPco (raw) & 406 & {$[45]$} \\
HiPco (purified) & 602 & {$[45]$} \\
HiPco & 498 & {$[49]$} \\
Carbolex (raw) & 380 & {$[50]$} \\
Carbolex (purified) & 547 & {$[50]$} \\
Shenzhen Nanotech (virgin) & 448 & {$[51]$} \\
Shenzhen Nanotech (annealed) ${ }^{\mathrm{b}}$ & 592 & {$[51]$} \\
Shenzhen Nanotech (virgin) & 603 & This work \\
Shenzhen Nanotech (oxidized) & 565 & This work \\
a Data extrapolated at $10{ }^{\circ} \mathrm{C} \mathrm{min}$ & -1 from original data at $5{ }^{\circ} \mathrm{C} \mathrm{min}^{-1}$. \\
b Vacuum annealing at $1000{ }^{\circ} \mathrm{C}$ for $2 \mathrm{~h}$. & &
\end{tabular}

approximately. We can therefore conclude that the FWCNTs used in this work have almost no amorphous carbon, are thermally stable with low number of defects, and the proposed mild oxidation treatment slightly reduces its thermal stability.

The slight reduction in thermal stability is in accord with the results of Yun and col. [38] but not sufficiently explained in the literature. One possible explanation considers that oxidation process starts with decarboxylation and $\mathrm{CO}_{2}$ generation creating a hole in the graphene sheet and inducing a cascade of events that ultimately collapses the carbon structure and its combustion. The presence of a higher amount of carboxylic groups facilitates initiation of the structure collapse and accelerates the combustion but should not change the overall mechanism. Therefore, the combustion mechanism should present the same overall activation energy.

There are several proposed methods to recover the oxidation activation energy from non-isothermal thermograms $[45,46,49,51,52]$. Chang's method [52] recovers $\Delta E$ from the slope of $\ln \left(q \cdot \mathrm{d} \alpha / \mathrm{d} T /(1-\alpha)^{n}\right)$ vs. $(1 / T)$ plot, where the conversion degree is defined as $\alpha=\left(m_{0}-m_{\mathrm{t}}\right) /\left(m_{0}-m_{\infty}\right), q$ is the heating rate and $\mathrm{d} \alpha / \mathrm{d} t=\mathrm{d} \alpha / \mathrm{d} T \cdot \mathrm{d} T / \mathrm{d} t=q \cdot \mathrm{d} \alpha / \mathrm{d} T$. Assuming that the oxidation of CNTs can be described by a single chemical process with a reaction order $n=1$, and that the temperature dependence of the reaction constant is described by the Arrhenius law within the narrow temperature range in which oxidation reaction takes place (roughly $150{ }^{\circ} \mathrm{C}$ ), apparent activation enthalpies could be obtained. In the conversion range $\alpha \in(0.05-0.9)$, we have found linear fits with regression coefficients higher than 0.993, as depicted in Fig. 6.

The recovered apparent activation enthalpies are $226 \mathrm{~kJ} \mathrm{~mol}^{-1}$ for as-received FWCNTs and $203 \mathrm{~kJ} \mathrm{~mol}^{-1}$ for oxidized FWCNTs. These values are notably higher than those previously reported in the literature $[47,49]$ which range between 120 and $140 \mathrm{~kJ} \mathrm{~mol}^{-1}$, and the difference of activation energies between the pristine and oxidized nanotubes can be hardly explained. However, a detailed analysis of the fitting could be carried out by studying the residuals.

As revealed in Fig. 6, reasonably good regression coefficients were obtained using Chang's method because the experimental "wavy" trend compensates positive and negative deviations, but residuals clearly show that experimental data follow a more complex kinetic behavior than initially assumed. All attempts to fit data to more complex single kinetic models, using either differential or integral features, similar to those reported by Vignes [45] or Illeková [49], failed, thus reflecting that no single model can explain accurately the oxidation kinetics of these FWCNTs. Therefore, in order to obtain model independent activation enthalpies, $T_{\mathrm{m}}$ was obtained at two other heating rates $\left(1\right.$ and $5{ }^{\circ} \mathrm{C} \min ^{-1}$ ), and the Kissinger method was applied [53,54]. Plots of $\ln \left(q / T_{\mathrm{m}}^{-2}\right)$ vs. $1 / T_{\mathrm{m}}$ allowed us to recover very similar activation energy values for both samples: 

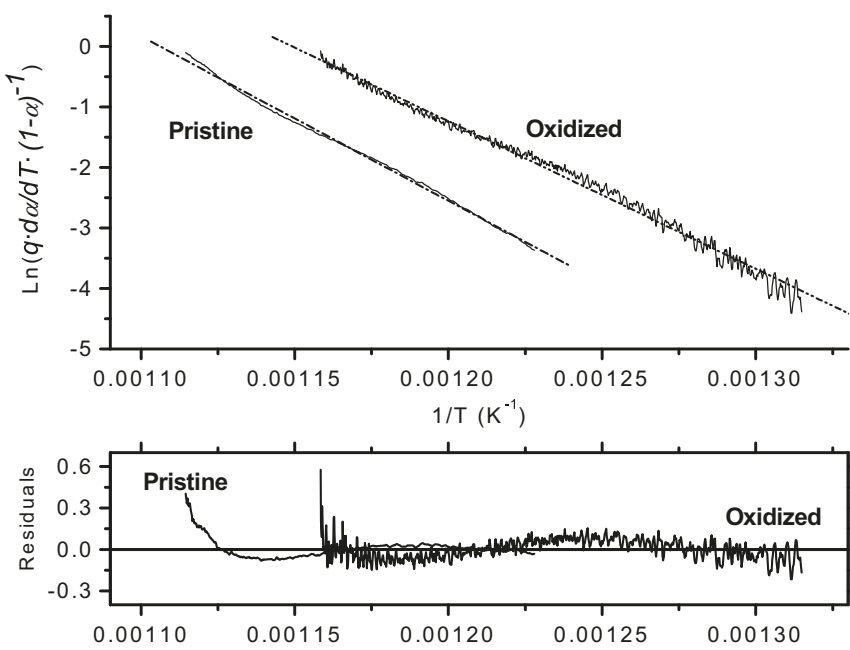

Fig. 6. Top: Chang's plot assuming reaction order $n=1$; Bottom: fitting residuals calculated according to $\left(O_{\mathrm{F}}-O_{\mathrm{O}}\right) / O_{\mathrm{O}}^{1 / 2}$ where $O_{\mathrm{F}}$ and $O_{\mathrm{O}}$ are the fitted and observed data respectively.

$\Delta E_{\text {pristine }}=138 \pm 1 \mathrm{~kJ} \mathrm{~mol}^{-1}$, and $\Delta E_{\text {oxidized }}=144 \pm 6 \mathrm{~kJ} \mathrm{~mol}^{-1}$. These values are in the same range as those reported in the literature, and allow us to conclude that the oxidation treatment studied in this paper increases reactivity of the tubes but do not modify neither their structure nor their combustion mechanism.

\section{Conclusions}

The yield of functionalized CNTs and the density of functional groups, ideally carboxylic acids that result from the oxidation process are important parameters. Many researchers have tried to explain the oxidation mechanism of CNTs, and it is believed that the chemical modifications of CNTs originate from defects sites, i.e., point defects, atomic impurities or heptagon-pentagon pairs that are under strain. In the presence of strong oxidizing agents, the graphitic structure is also attacked, thus creating a large number of unwanted defect sites that could react with the oxidizing agent.

The oxidation method studied in this work avoids the use of unpleasant strong acids and uses UV light to induce hydrogen peroxide photolysis thus creating strong oxidant $\mathrm{OH}^{*}$ radicals that adsorb on preferred sites and oxidize other pre-existing oxidized forms of carbon. The generated carboxylic groups partially originate from oxidation of lower oxidation states, such as carbonyl groups, initially present and probably due to an acid purification process. Fenton oxidation parallel route may play a role on the functionalization of FWCNT from Shenzhen, but it can be excluded in the oxidation of GS and NCL nanotubes. Whether the carboxylic groups are uniformly distributed along the tube walls or forming small clusters is an issue under current investigation. In any case, the yield of this reaction appeared to be higher than those reported in the literature using other methods and can be also used with MWCNT as confirmed by our results on GS and NCL nanotubes. The method reported here appears to be efficient with no apparent structural damage on the tubes, it slightly decreases the thermal stability of the tubes, it increases dispersibility and the resulting carboxylized FWCNTs could now be more efficiently dispersed in liquid media and incorporated in polymer composites.

\section{Acknowledgments}

O. Martín and J. Baselga acknowledge support from Ministerio de Ciencia e Innovación (Spain) under grants MAT2010-17091 and
MAT2009-06296E. M. Terrones acknowledges support from the Research Center for Exotic Nanocarbons, Japan regional Innovation Strategy Program by the Excellence, JST. T. Blanco acknowledges support from CDTI under the project NANOMULFUN (SAE20081035).

\section{Appendix A. Supplementary data}

Supplementary data related to this article can be found at http:// dx.doi.org/10.1016/j.matchemphys.2013.03.060.

\section{References}

[1] H.S. Nalwa, Handbook of Nanostructured Materials and Nanotechnology, vol 5, Academic Press, New York, 2000.

[2] H. Cebeci, R. Guzman de Villoria, A.J. Hart, B.L. Wardle, Compos. Sci. Technol. $69(15-16)$ (2009) 2649-2656.

[3] J.N. Coleman, U. Khan, W.J. Blau, Y.K. Guńko, Carbon 44 (9) (2006) 1624-1652.

[4] W. Bauhofer, J.Z. Kovacs, Compos. Sci. Technol. 69 (2009) 1486-1498.

[5] E.T. Thostenson, C. Li, T.-W. Chou, Compos. Sci. Technol. 65 (2005) 491-516.

[6] V. Georgakilas, K. Kordatos, M. Prato, D.M. Guldi, M. Holzinger, A. Hirsch, J. Am. Chem. Soc. 124 (2002) 760-761

[7] B. Fernandez-d'Arlas, S. Goyanes, G.H. Rubiolo, I. Mondragon, M.A. Corcuera, A. Eceiza, J. Nanosci. Nanotechnol. 9 (2009) 6064-6071.

[8] S. Meuer, L. Braun, R. Zentel, Macromol. Chem. Phys. 210 (2009) 1528-1535

[9] T. Ramanathan, F.T. Fisher, R.S. Ruoff, L.C. Brinson, Chem. Mater. 17 (2005) 1290-1295.

[10] L. Shao, Y. Bai, X. Huang, Z. Gao, L. Meng, Y. Huang, J. Ma, Mater. Chem. Phys 116 (2009) 323-326.

[11] J.L. Bahr, J. Yang, D.V. Kosynkin, M.J. Bronikowski, R.E. Smalley, J.M. Tour, J. Am. Chem. Soc. 123 (2001) 6536-6542.

[12] B.B. Parekh, G. Fanchini, G. Eda, M. Chhowalla, Appl. Phys. Lett. 90 (2007) 121913.

[13] H. Tantang, J.Y. Ong, C.L. Loh, X. Dong, P. Chen, Y. Chen, X. Hu, L.P. Tan, L.J. Li, Carbon 47 (2009) 1867-1885.

[14] H.-Z. Geng, K.K. Kim, K.P. So, Y.S. Lee, Y. Chang, Y.H. Lee, J. Am. Chem. Soc. 129 (2007) 7758-7759.

[15] Y.J. Kim, T.S. Shin, H.D. Choi, J.H. Kwon, Y.C. Chung, H.G. Yoon, Carbon 43 (2005) $23-30$

[16] X. Liang, L. Ling, C. Lu, L. Liu, Mater. Lett. 43 (2000) 144-147.

[17] C. Wang, G. Zhou, J. Wu, B.L. Gu, W. Duan, Appl. Phys. Lett. 89 (2006) 173130.

[18] R. Graupner, J. Abraham, A. Vencelová, T. Seyller, F. Hennrich, M.M. Kappes, A. Hirsch, L. Ley, Phys. Chem. Chem. Phys. 5 (2003) 5472-5476.

[19] Y. Yang, H. Zou, B. Wu, Q. Li, J. Zhang, Z. Liu, X. Guo, Z. Du, J. Phys. Chem. B 106 (2002) 7160-7162.

[20] J. Liu, A.G. Rinzler, H. Dai, J.H. Hafner, R.K. Bratley, P.J. Boul, A. Lu, T. Iverson, K. Shelimov, C.B. Huffman, F. Rodriguez-Macias, Y.S. Shon, T.R. Lee, D.T. Colbert, R.E. Smalley, Science 280 (1998) 1253-1256.

[21] A. Rasheed, J.Y. Howe, M.D. Dadmun, P.F. Britt, Carbon 45 (2007) 1072-1080

[22] V. Datsyuk, M. Kalyva, K. Papagelis, J. Parthenios, D. Tasis, A. Siokou, I. Kallitsis, C. Galiotis, Carbon 46 (2008) 833-840.

[23] P.V. Lakshminarayanan, H. Toghiani, C.U. Pittman, Carbon 42 (2004) 2433-2442.

[24] I.D. Rosca, F. Watari, M. Uo, T. Akasaka, Carbon 43 (2005) 3124-3131.

[25] A.B. González-Guerrero, E. Mendoza, E. Pellicer, F. Alsina, C. Fernandez-Sanchez, L.M. Lechuga, Chem. Phys. Lett. 462 (2008) 256-259.

[26] M. Holzinger, A. Hirsch, P. Bernier, G.S. Duesberg, M. Burghard, Appl. Phys. A 70 (2000) 599-602.

[27] H. Naeimi, A. Mohajeri, L. Moradi, A.M. Rashidi, Appl. Surf. Sci. 256 (2009) 631-635.

[28] L. Cai, J.L. Bahr, Y. Yao, J.M. Tour, Chem. Mater. 14 (2002) 4235-4241.

[29] E. Najafi, J.Y. Kim, S.H. Han, K. Shin, Colloids Surf. A 284-285 (2006) 373-378

[30] W. Li, Y. Bai, Y. Zhang, M. Sun, R. Cheng, X. Xu, Y. Chen, Y. Mo, Synth. Met. 155 (2005) 509-515.

[31] K. Hernadi, A. Siska, L. Thiên-Nga, L. Forró, I. Kiricsi, Solid State Ion. 141-142 (2001) 203-209.

[32] A. Kuznetsova, I. Popova, J.T. Yates, M.J. Bronikowski, C.B. Huffmam, J. Liu, R.E. Smalley, H.H. Hwu, J.G. Chen, J. Am. Chem. Soc. 123 (2001) 10699-10704.

[33] S. Bose, R.A. Khare, P. Moldenaers, Polymer 51 (2010) 975-993.

[34] L.I. Nasibulina, I.V. Anoshkin, A.G. Nasibulin, A. Cwirzen, V. Penttala, E.I. Kauppinen, J. Nanomater. 2012 (2012) 169262.

[35] J. Zhang, H. Zou, Q. Qing, Y. Yang, Q. Li, Z. Liu, X. Guo, Z. Du, J. Phys. Chem. B 107 (2003) 3712-3718.

[36] K. Peng, L.-Q. Liu, H. Li, H. Meyer, Z. Zhang, Carbon 49 (2011) 70-76.

[37] G.L. Luque, M.I. Rojas, G.A. Rivas, E.P.M. Leiva, Electrochim. Acta 56 (2010) 523-530.

[38] Y. Peng, H. Liu, Ind. Eng. Chem. Res. 45 (2006) 6483-6488.

[39] M. Muruganandham, M. Swaminathan, Dyes Pigments 62 (2004) 269-275.

[40] C.G. Salzmann, S.A. Llewellyn, G. Tobias, M.A.H. Ward, Y. Huh, M.L.H. Green, Adv. Mater. 19 (2007) 883-887.

[41] R. Verdejo, S. Lamoriniere, B. Cottam, A. Bismarck, M. Shaffer, Chem. Commun. 5 (2007) 513-515. 
[42] Z. Wang, M.D. Shirley, S.T. Meikle, R.L.D. Whitby, S.V. Mikhalovsky, Carbon 47 (2009) 73-79.

[43] J.H. Bae, A.M. Shanmugharaj, W.H. Noh, W.S. Choi, S.H. Ryu, Appl. Surf. Sci. 253 (2007) 4150-4155.

[44] H. Hu, A. Yu, E. Kim, B. Zhao, M.E. Itkis, E. Bekyarova, R.C. Haddon, J. Phys. Chem. B 109 (2005) 11520-11524.

[45] A. Vignes, O. Dufaud, L. Perrin, D. Thomas, J. Bouillard, A. Janès, C. Vallières, Chem. Eng. Sci. 64 (2009) 4210-4221.

[46] S. Arepalli, P. Nikolaev, O. Gorelik, V.G. Hadjiev, W. Holmes, B. Files, L. Yowell, Carbon 42 (2004) 1783-1791.

[47] R. Brukh, S. Mitra, J. Mater. Chem. 17 (2007) 619-623.

[48] A.M.F. Lima, A.W. Musumeci, H.-W. Liu, E.R. Waclawik, G.G. Silva, J. Therm. Anal. Calorim. 97 (2009) 257-263.

[49] E. Illeková, K. Csomorová, J. Therm. Anal. Calorim. 80 (2005) 103-108.

[50] B. Vigolo, C. Hérold, J.-F. Marêché, J. Ghanbaja, M. Gulas, F. Le Normand, R. Almairac, L. Alvarez, J.-L. Bantignies, Carbon 48 (2010) 949-963.

[51] D.K. Singh, P.K. Iyer, P.K. Giri, J. Appl. Phys. 108 (2010) 084313.

[52] S. Sarkar, P.K. Das, S. Bysakh, Mater. Chem. Phys. 125 (2011) 161-167.

[53] D.K. Chattopadhyay, D.C. Webster, Prog. Polym. Sci. 34 (2009) 1068-1133.

[54] H.E. Kissinger, Anal. Chem. 29 (1957) 1702-1706. 